\title{
Predicting outcomes after acute reperfusion therapy for basilar artery occlusion
}

\author{
Jadhav, A. P.
}

2020-11

Jadhav , A P , Desai , S M , Panczykowski , D M , Rangaraju , S , Campbell , D , Ritvonen , J K, Schreiner , M , Silvennoinen , H, Gerber , J, Puetz , V , Raza , S A, Haussen , D C , Nogueira, R G , Strbian , D , Jovin , T G \& Lindsberg, P J 2020 , ' Predicting outcomes after acute reperfusion therapy for basilar artery occlusion ', European Journal of Neurology , vol. 27 , no. 11 , pp. 2176-2184 . https://doi.org/10.1111/ene.14406

http://hdl.handle.net/10138/333733

https://doi.org/10.1111/ene.14406

acceptedVersion

Downloaded from Helda, University of Helsinki institutional repository.

This is an electronic reprint of the original article.

This reprint may differ from the original in pagination and typographic detail.

Please cite the original version. 


\section{Predicting Outcomes After Acute Reperfusion Therapy for Basilar Artery Occlusion}

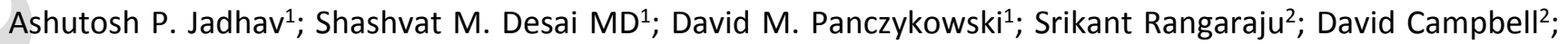
Juhani K Ritvonen³; Monique Schreiner4; Heli Silvennoinen³; Johannes Gerber5; Volker Puetz"; Syed Ali Raza²; Diogo C. Haussen²; Raul G. Nogueira²; Daniel Strbian³; Tudor G Jovin ${ }^{6}$; Perttu J. Lindsberg ${ }^{3}$

Title: -- 80 characters (with spaces)

Running Title: POST-VB Score

Abstract: --250 words

Manuscript: --3043 words

References: 23

Figures: 5

Tables: 1

Key Words: Acute Stroke; CT; Diffusion-Weighted Imaging; MRI; Stroke Recovery; Thrombolysis; Transient Ischemic Attack

Abbreviation used in this paper: AIS (acute ischemic stroke), ASPECTS (Alberta stroke program early CT score), CT (computed tomography), emergency department (ED), LVO (large vessel occlusion), NIHSS (National Institutes of Health Stroke Scale), $\mathrm{PCl}$ (percutaneous coronary intervention)

Author contributions to the study and manuscript preparation include the following: Conception and design: Jadhav, Jovin, Lindsberg. Acquisition of data: all authors. Analysis and interpretation of data: Jadhav, Kenmuir, Rangaraju, Panczykowski, Desai, Strbian. Drafting the article: Jadhav, Panczykowski, Desai. Critically revising the article: all authors. Administrative/technical/material support: all authors. Study supervision: Jovin.

Statistical Analysis: Shashvat M Desai MD, Department of Neurology, University of Pittsburgh, Pittsburgh, PA, USA

Affiliation:

University of Pittsburgh, Departments of Neurology and Neurosurgery1, Pittsburgh, PA, USA; Department of Neurology, Emory University and Grady Memorial Hospital2, Atlanta, GA, USA; Departments of Neurology and Radiology, Helsinki University Hospital3, Helsinki, Finland; Department of Neurology and and Clinical Neurosciences ${ }^{4}$ and Institute of Neuroradiology 5 , Carl Gustav Carus University Hospital, Dresden, Germany; Department of Neurology, Cooper University Hospital, Camden NJ, USA ${ }^{6}$

Correspondence to: Perttu J. Lindsberg, Neurocenter, Neurology, Helsinki University Hospital, PO Box 700, Haartmaninkatu 8, 00029 HUS, Helsinki, Finland, Email perttu.lindsberg@hus.fi

This article has been accepted for publication and undergone full peer review but has not been through the copyediting, typesetting, pagination and proofreading process, which may lead to differences between this version and the Version of Record. Please cite this article as doi: $\underline{10.1111 / \text { ENE.14406 }}$

This article is protected by copyright. All rights reserved 
Study Funding: None

Search Terms: Stroke [2], Infarction [6], Other cerebrovascular diseases/ stroke [13], CT [119], MRI [120]

Sources of Funding: none

Disclosure: none

Informed consent: Not required due to retrospective nature of study

This article is protected by copyright. All rights reserved 
DR. SHASHVAT M DESAI (Orcid ID : 0000-0001-9013-6929)

DR. JUHANI RITVONEN (Orcid ID : 0000-0003-0922-2358)

DR. JOHANNES CHRISTOPH GERBER (Orcid ID : 0000-0001-7465-8700)

DR. DIOGO C. C. HAUSSEN (Orcid ID : 0000-0003-1884-2196)

Article type : Original Article

\section{Predicting Outcomes After Acute Reperfusion Therapy for Basilar Artery Occlusion}

Ashutosh P. Jadhav ${ }^{1}$; Shashvat M. Desai MD; David M. Panczykowski ${ }^{1}$; Srikant Rangaraju ${ }^{2}$; David Campbell $^{2}$; Juhani K Ritvonen ${ }^{3}$; Monique Schreiner ${ }^{4}$; Heli Silvennoinen ${ }^{3}$; Johannes Gerber ${ }^{5}$; Volker Puetz ${ }^{4}$; Syed Ali Raza ${ }^{2}$; Diogo C. Haussen²; Raul G. Nogueira²; Daniel Strbian ${ }^{3}$; Tudor G Jovin ${ }^{6}$; Perttu J. Lindsberg ${ }^{3}$ Title: -- 80 characters (with spaces)

Running Title: POST-VB Score

Abstract: --250 words

Manuscript: --3043 words

References: 23

Figures: 5

Tables: 1

Key Words: Acute Stroke; CT; Diffusion-Weighted Imaging; MRI; Stroke Recovery; Thrombolysis; Transient Ischemic Attack

Abbreviation used in this paper: AIS (acute ischemic stroke), ASPECTS (Alberta stroke program early CT score), CT (computed tomography), emergency department (ED), LVO (large vessel occlusion), NIHSS (National Institutes of Health Stroke Scale), PCl (percutaneous coronary intervention)

This article is protected by copyright. All rights reserved 
Author contributions to the study and manuscript preparation include the following: Conception and design: Jadhav, Jovin, Lindsberg. Acquisition of data: all authors. Analysis and interpretation of data: Jadhav, Kenmuir, Rangaraju, Panczykowski, Desai, Strbian. Drafting the article: Jadhav, Panczykowski, Desai. Critically revising the article: all authors. Administrative/technical/material support: all authors. Study supervision: Jovin.

Statistical Analysis: Shashvat M Desai MD, Department of Neurology, University of Pittsburgh, Pittsburgh, PA, USA

Affiliation:

University of Pittsburgh, Departments of Neurology and Neurosurgery1, Pittsburgh, PA, USA; Department of Neurology, Emory University and Grady Memorial Hospital2, Atlanta, GA, USA; Departments of Neurology and Radiology, Helsinki University Hospital3, Helsinki, Finland; Department of Neurology and and Clinical Neurosciences ${ }^{4}$ and Institute of Neuroradiology ${ }^{5}$, Carl Gustav Carus University Hospital, Dresden, Germany; Department of Neurology, Cooper University Hospital, Camden NJ, USA ${ }^{6}$

Correspondence to: Perttu J. Lindsberg, Neurocenter, Neurology, Helsinki University Hospital, PO Box 700, Haartmaninkatu 8, 00029 HUS, Helsinki, Finland, Email perttu.lindsberg@hus.fi

Study Funding: None

Search Terms: Stroke [2], Infarction [6], Other cerebrovascular diseases/ stroke [13], CT [119], MRI [120] Sources of Funding: none

Disclosure: none

Informed consent: Not required due to retrospective nature of study

Data sharing: Data used to prepare this manuscript may be made available upon reasonable request to the corresponding author

This article is protected by copyright. All rights reserved 


\begin{abstract}
Background: Basilar artery occlusion (BAO) leads to high rates of morbidity and mortality, despite successful recanalization. The discordance between flow restoration and long-term functional status clouds clinical decision making regarding further aggressive care. We sought to develop and validate a practical, prognostic tool for the prediction of 3-month favorable outcome after acute reperfusion therapy for BAO.
\end{abstract}

Methods: This retrospective, multicenter, observational study was conducted at four high-volume stroke centers in the U.S. and Europe. Multivariate regression analysis was performed to identify predictors of favorable outcome (90-day mRS scores 0-2) and derive a clinically applicable prognostic model (the Pittsburgh Outcomes after Stroke Thrombectomy-VertebroBasilar (POST-VB) score). The POST-VB score was evaluated and internally validated apropos of calibration and discriminatory ability. External validity was assessed in patient cohorts at 3 separate centers.

Results: In the derivation cohort of 59 patients, independent predictors of favorable outcome included smaller brainstem infarct volume on post-procedure MRI $(p<0.01)$ and younger age $(p=0.01)$. The POSTVB score was calculated as: age $+(10 \times$ brainstem infarct volume $)$. The POST-VB score demonstrated excellent discriminatory ability $(A \cup C=0.91)$ and adequate calibration $(p=0.88)$ in the derivation cohort (center A). POST-VB performed equally well across the 3 external validation cohorts (center $B$, $A \cup C=0.89$; center $C, A \cup C=0.78$; center $D, A \cup C=0.80$ ). Overall, a POST-VB score $<49$ was associated with an $88 \%$ likelihood of favorable outcome, as compared to $4 \%$ with a score $\geq 125$.

Conclusions: The POST-VB score effectively predicts 3-month functional outcome following acute reperfusion therapy for $\mathrm{BAO}$ and may aid in guiding post-procedural care.

This article is protected by copyright. All rights reserved 


\section{INTRODUCTION}

Basilar artery occlusion (BAO) may result in devastating ischemia of critical anatomic structures within the brainstem and cerebellum, and if left untreated is associated with a dismal prognosis and high mortality ( $\geq 90 \%)$. $(1,2)$ Recently concluded randomized controlled trials, the BEST and the BASICS trial, did not demonstrate a benefit of endovascular thrombectomy (EVT) over best medical management for acute basilar artery occlusion (BAO) strokes.(1) Retrospective data suggests that endovascular recanalization for the treatment of BAO maybe safe and effective, with successful reperfusion being achieved in $81-92 \%$ of patients. $(3,4)$ Intravenous thrombolytic therapy (IVT) has led to at least as good outcomes as the endovascular approach.(5,6) Despite the high rate of recanalization with endovascular treatment, the majority of patients intervened upon still experience poor outcomes (90-day mRS $\geq 3$ in $55-68 \%) \cdot(3,4,7,8)$

With increasing adoption of aggressive interventions for BAO, studies have sought to elucidate the potential capabilities of pre-procedural characteristics to predict functional outcome and aid in the selection of patients most likely to benefit from recanalization.(9-11) Although the prognostic value of overall infarct volume is well established in anterior circulation stroke, initial analyses evaluating BAO did not find similar correlations with posterior circulation lesions.(12) However, recent trials incorporating pathoanatomic scoring of posterior circulation infarction have demonstrated improved correlation with functional outcome after BAO. $(9,12,13)$ Nevertheless, even in highly selected analyses (i.e. those excluding patients with extensive brainstem ischemic changes) favorable outcomes following successful recanalization may still only be observed in $49 \%$ of BAO patients.(3) Conversely, utilizing overly selective pre-procedural criteria may increase the risk of withholding therapy from patients with a smaller yet clinically important potential for benefit.

Early and prolonged invasive supportive measures are frequently necessary in patients destined for good outcomes, thus forcing decisions regarding aggressiveness of care to be made in the immediate post-procedure period. Incongruent rates between successful recanalization and good clinical outcome necessitates accurate prognostic tools to better discriminate between those patients who will benefit from early and aggressive supportive measures and those who should be considered for de-escalation of care. No study has yet assessed and/or externally validated post-procedural characteristics as a means of better predicting the functional outcome of those who have undergone acute reperfusion therapy of $\mathrm{BAO}$. We realize that all decisions of $\mathrm{BAO}$ build on rapid triage of candidates for increasingly accessible

This article is protected by copyright. All rights reserved 
recanalization therapy. Not too infrequently, this results in still critically ill patients with unequivocal prognosis, in whom post procedure phase requires careful weighing of optimal care, i.e. continuation of aggressive measures or rather de-escalation of advanced care. A prognostic score, with the ability to predict functional outcome at 3 months based on post procedure information, may be an important tool to support decision making for a longer-term therapy plan.

The purpose of this study was to develop, evaluate, and externally validate the utility of a postprocedural scoring system for the prediction of functional outcome after acute reperfusion therapy of BAO.

This article is protected by copyright. All rights reserved 


\section{METHODS}

\section{Patient Population}

This retrospective, multicenter, observational study was conducted at four high-volume stroke centers in the USA (Pittsburgh-Center A, Atlanta-Center B), Germany (Dresden-Center C), and Finland (HelsinkiCenter D) with IRB (University of Pittsburgh Ethics Board) or local ethics committee approval. Informed consent was not required due retrospective analysis of the data). The data used for this study are available from the corresponding author upon reasonable request. The overall study sample was created by retrospectively identifying all consecutive patients with radiographically confirmed acute symptomatic BAO between July 2000 to July 2012. Patients were included for analysis if presenting neurologic symptomatology was referable to BAO as demonstrated on conventional catheter-based digital subtraction angiography (DSA) or MR/CT angiography, and followed by post-procedural MR imaging. BAO was defined as complete absence of anterograde flow in the basilar artery despite delayed filming. Patients were excluded from analysis if clinical or radiographic data were missing or incomplete. Baseline demographic information, clinical characteristics at presentation, and clinical course were all prospectively recorded.

\section{Patient Selection and Treatment}

In accordance with current guidelines intravenous recombinant tissue plasminogen activator (IV tPA) was administered to eligible patients. Patients were deemed to be candidates for endovascular therapy per local institutional protocols, broadly following criteria: 1) clinical symptoms referable to posterior circulation ischemia, and 2) pre-treatment imaging without evidence of intracranial hemorrhage and/or extensive infarction that precluded a meaningful recovery in the opinion of the treating stroke neurologist (i.e. entirety of the pons or bilateral thalami). The decision to proceed with endovascular treatment was made between the stroke neurologist and neuro-interventionalist. The endovascular reperfusion techniques utilized included intra-arterial pharmacologic thrombolysis and/or mechanical thrombectomy. The technical approach was left to the discretion of the treating neurointerventionalist. The details of these endovascular techniques have been extensively described elsewhere.(14,15)

\section{Predictive Characteristics}

Baseline demographics, clinical stroke severity, procedural characteristics, angiographic reperfusion status, and final infarct burden on post-procedural MR imaging were all assessed as potential prognostic

This article is protected by copyright. All rights reserved 
variables. Clinical stroke severity was measured by NIHSS score. Reperfusion status was assessed on final angiographic runs according to the modified Thrombolysis in Cerebral Infarction (mTICl) scale; successful recanalization was defined as $\mathrm{mTICl}$ grade $2 \mathrm{~b}$ or 3.(16) Final infarct burden was determined by post-procedural MRI that was performed within 24-hours of the procedure. MRI was performed on 1.5 or 3 Tesla scanners according to institutional protocols. At a minimum, all centers performed these sequences: 1) T2-weighted gradient-echo, 2) diffusion-weighted imaging with apparent diffusion coefficient map reconstruction; and 3) fluid-attenuated inversion recovery. Sectorial volumetric analysis of post-procedure infarct burden assessed the following anatomic regions irrigated by the posterior circulation: each thalamus, each occipital lobe, brainstem (midbrain, pons and medulla), and cerebellum [Figure 1]. At Center A, C and D, the infarct volume for each anatomic sector was calculated by outlining the DWI lesion perimeter with a graphing tool available on the Siemens workstation to determine infarct area on each axial slice. At center B, area measurement for each slice was performed manually (previously proven to have high correlation with Siemens graphing tool).(17) The product of the slice thickness and the summation of infarct areas gave the infarct volume for each anatomic sector.

\section{Outcomes}

The primary outcome was the degree of functional recovery at 3-month follow-up visit as determined by a trained, unblinded stroke neurologist according to the modified Rankin Scale (mRS), and was recorded prospectively. Favorable functional recovery was defined as a mRS of 0 to 2 .

\section{Statistical Analysis}

Acceptable Type I error was set $a$ priori at $\alpha=0.05$ for all statistical tests. All analyses were defined $a$ priori. Patients with missing and/or incomplete clinical, radiographic, or outcome data were excluded from analyses. Continuous demographic characteristics were assessed for normality using the Kolmogorov-Smirnov test; normally distributed data were analyzed by t-test while the remainders were compared using the Wilcoxon Rank-Sum test. Categorical data were analyzed by Pearson's chi-squared or Fisher's exact test.

Initial univariable and multivariate analysis and predictive model derivation was performed utilizing the UPMC (center A) cohort. Univariable regression analysis was performed to assess the association between predictive variables and 3-month favorable functional outcome. Multivariable regression analysis was performed with stepwise backwards elimination to fit a predictive model; following 
adjustment, variables that did not reach significance at the $\alpha=0.10$ level were excluded. A scoring system for the prediction of functional outcome (the Pittsburgh Outcomes after Stroke Thrombectomy VertebroBasilar (POST-VB)) was developed based on $\beta$-coefficients of each specific predictive variable from the fitted multivariate logistic regression model.

We assessed the performance of the POST-VB apropos of accuracy, discrimination, and calibration. Accuracy was assessed through calculation of the average prediction error, or Brier score; ideally scores should be $<0.25$ (better than flip of a coin). Discrimination was assessed using the area under the receiver operating characteristic curve (AUC). Using current statistical consensus, AUC of 0.8 to 0.9 are considered very good, 0.7 to 0.8 as adequate, and below 0.7 as poor.(18) Calibration is the measurement of agreement between predicted and observed risk over the full range of predicted probabilities, and was tested with the Hosmer-Lemeshow goodness-of-fit test. To perform this test patients were grouped by POST-VB score into 5 levels and significant deviation was set $a$ priori at $\alpha$ $\leq 0.05$. The evaluation of predictive performance using patients from the original derivation sample may result in an overly optimistic estimate of predictive performance, therefore a 10-fold cross validation for the AUC was applied.(19) Clinical utility was evaluated by decision curve analysis.(20,21)

External validity of the POST-VB scoring system was assessed in separate cohorts of patients suffering BAO undergoing acute reperfusion therapy at 3 high-volume stroke centers (Atlanta, Dresden, and Helsinki); these retrospective analyses were performed on the prospectively collected data from each site. Predictive performance of the POST-VB score applied to all external cohorts was similarly assessed apropos of discrimination and calibration as described above.

Means are reported as mean \pm standard deviation; significant associations between outcomes and predictors are reported as odds ratios (OR) with 95\% confidence intervals ( $\mathrm{Cl}$ ). Data were analyzed using Stata 14 (College Station, TX).

This article is protected by copyright. All rights reserved 


\section{RESULTS}

Baseline demographics and clinical characteristics for the 59 patients analyzed in the derivation cohort (patients from center $A$ ) are displayed in Table 1. The mean age was $64 \pm 13$ years; $34 \%$ of this cohort were female. The median NIHSS on presentation was 18 (IQR 22), and 48\% presented with an NIHSS $\geq 20$.

Twelve-percent of the patients presenting to center $A$ met criteria for and subsequently received IV tPA. Median time from symptom onset to initiation of endovascular treatment was 13 hours (IQR 35). Endovascular techniques utilized for recanalization (either alone or in combination) included intraarterial pharmacologic thrombolysis (42\%), mechanical thrombectomy (including $\mathrm{MERCl}$ retriever, stentriever, or manual aspiration), and/or angioplasty with/without stenting; proportions of individual techniques are displayed in Table 1. Successful reperfusion ( $\mathrm{mTICl} 2 \mathrm{~b}-3$ ) was achieved in $90 \%$ of cases.

Figure 2 displays mean final infarct volumes by anatomic region, as demonstrated on post-procedural MRI. Thirty-seven percent of patients had evidence of infarction in all anatomic regions assessed. Overall, the median total infarct volume was $26 \mathrm{~mL}$ (IQR 46, range 0-295).

\section{Outcomes and Predictive Model Derivation}

Overall, $56 \%$ of patients survived beyond 3 months. Thirty-two percent of the entire cohort achieved functional independence (mRS 0-2). Figure S1 depicts a breakdown of the derivation cohort by 3-month mRS. Baseline demographics, clinical characteristics, procedural factors, and post-procedural imaging findings were assessed for association with 3-month functional outcome utilizing univariable logistic regression. The factors significantly independently associated with 3-month functional independence included smaller infarct volumes within the brainstem (OR 2.02; 95\% $\mathrm{Cl} 1.35-3.01$ ) and cerebellum (OR 1.04; $95 \% \mathrm{Cl} 1.01-1.07)$. Stepwise, multivariable logistic regression analysis identified younger age and smaller brainstem infarct volume as significant independent predictors of 3-month functional independence (OR 1.04; 95\% 1.01-1.07 and OR 6.57; 95\% Cl 1.76-24.5, respectively). The equation for the scoring system for the prediction of functional outcome was derived by incorporating the $\beta$ coefficients of these independently significant variables from this multivariable logistic regression model (POST-VB score $=$ age $+10 \times$ brainstem infarct volume).

\section{Predictive Model Assessment}

This article is protected by copyright. All rights reserved 
The POST-VB score for the prediction of 3-month functional independence displayed adequate accuracy (Brier score $=0.15)$. The ability of the POST-VB scoring system to discriminate between patients achieving long-term functional independence versus those being severely disabled or dead, as measured by the AUC curve was $0.91(95 \% \mathrm{Cl} 0.84-0.98)$ indicating excellent discriminatory ability. The POST-VB score displayed effective calibration for predicting functional independence (Hosmer-Lemeshow test, $p=0.88$ ). The clinical utility of POST-VB to predict who is most likely to achieve functional independence was evaluated through decision curve analysis as an extension of clinical decision-making based on history, exam, and hospital course. Use of the POST-VB score displayed superior net benefit as compared to proceeding with aggressive care in all patients beginning at threshold probability (i.e. perceived likelihood of functional recovery) of approximately $5 \%$ or higher. This correlated with a net reduction of inappropriately pursuing aggressive care in 11 patients likely to be severely disabled or dead for every 20 patients at a decision threshold of $50 \%$ (i.e. clinicians perceived likelihood of poor outcome).

The external validity of POST-VB score to predict 3-month favorable functional outcome was assessed by retrospective application to cohorts of patients undergoing endovascular treatment for BAO at 3 independent, high-volume stroke centers (center $B$, center $C$, and center $D$ ). Clinical characteristics used in the model were compared between derivation and validation cohorts; brainstem infarct volume (ml) was the only significantly different characteristic between cohorts (center $A \quad 4.6 \pm 4.7 \mathrm{ml}$, center $B$ $0.52 \pm 0.57 \mathrm{ml}$, center $C 1.3 \pm 2.0 \mathrm{ml}$, and center $D 3.1 \pm 3.6 \mathrm{ml}, \mathrm{p}<0.01)$. Within each cohort, age and brainstem infarct volume remained significant predictors of 3-month functional outcome in both univariate and multivariable regression analyses. Figure 3 displays the receiver operating characteristic plot for the POST-VB score applied at each center. The discriminatory ability of the POST-VB scoring system for predicting long-term functional independence ranged from good to very good, with AUCs of 0.89 for center $B, 0.78$ for center $C$, and 0.80 for center $D$. Rates of mRS $0-2$ and mRS $0-3$ at 90 days by POSTVB (Binned-0-49, 50-74, 75-99, 100-124, >124) scores are displayed in Figure S2.

This article is protected by copyright. All rights reserved 


\section{DISCUSSION}

The POST-VB scoring system is a simple and clinically applicable method able to reliably predict 3-month functional outcome following thrombectomy for acute BAO. This scoring system demonstrated very good discriminatory ability as well as external validity when applied to patients treated at a variety of high-volume stroke centers (center $B, A \cup C=0.89$; center $C, A \cup C=0.78$; center $D, A \cup C=0.80$ ).

Although BAO continues to carry a high mortality rate (approximately $30-40 \%$ ), up to 4 in 10 BAO stroke patients may achieve functional independence. The POST-VB score successfully discriminated between those patients who will benefit from early and aggressive supportive measures and those who may be considered for de-escalation of care. Overall, a POST-VB score $<49$ was associated with an $88 \%$ likelihood of favorable outcome, as compared to $4 \%$ with a score $\geq 125$. As a practical outcome the results of the clinical utility analyses revealed that applying the POST-VB score added significant clinical value above that currently offered by routine consideration of individual clinical factors, regardless of a treating physician's certainty of an outcome. Utilization of the POST-VB score for outcome prediction demonstrated a net benefit over standard practice beginning at a perceived certainty threshold (perceived likelihood of correctly predicting outcome) from as low as $5 \%$ and extending up to $100 \%$. Furthermore, decision analysis demonstrated that utilizing the POST-VB score would lead to reduced inappropriate utilization of aggressive care in 11 patients likely to be severely disabled or dead for every 20 patients at a decision threshold of $50 \%$ (i.e. physician certainty of outcome equal to flipping a coin).

As of yet there are no randomized controlled trial data supporting the efficacy of thrombectomy for the treatment BAO. The Basilar Artery Occlusion Chinese Endovascular Trial (BEST) was neutral and the Basilar Artery International Cooperation Study (BASICS) Trial results are eagerly awaited.(1,22) The current American Stroke Association 2019 guidelines offer a class Ilb recommendation for thrombectomy in BAO patients within 6 hours of onset. Despite a lack of definitive evidence, endovascular thrombectomy has been increasingly offered as a salvage operation based on promising results from case series. $(3,4,6,7)$ Despite advances in technique and recanalization rates as high as 90\%, the majority of patients intervened upon still experience poor outcomes. Previous studies have sought to determine which baseline characteristics are determinants of poor outcomes. Characteristics associated with poor outcome have included age, higher stroke severity in clinical and imaging work-up, longer time to recanalization, and incomplete or failed recanalization. $(4,5,8)$ The current study is the first to demonstrate that not only post-procedural infarct volume, but also location is an independent

This article is protected by copyright. All rights reserved 
predictor of outcome following BAO. Specifically, infarcts involving the brainstem portend a worse outcome.

The relationship between final infarct volume and functional outcome has been established in the case of anterior circulation stroke. Our group has previously reported that in a homogeneous group suffering middle cerebral artery occlusion (segment 1) treated with endovascular therapy, the most powerful predictors of outcome were age and final infarct volume. From this we developed and validated a similar scoring system for outcome prediction in anterior circulation stroke (Pittsburgh Outcomes After Stroke Thrombectomy (POST)), based on patient age, final infarct volume, and presence of hemorrhage.(17)

This current study supports a similar paradigm for outcome prediction in stroke secondary to BAO treated with endovascular therapy. However, unique to $\mathrm{BAO}$, these current results indicate that infarct location also plays a critical role in prediction. Our findings are in keeping with previously published analyses evaluating brainstem stroke burden utilizing a semi-quantitative approach. A scoring system based solely on brainstem infarct volume was utilized to predict presence and length of coma and outcome.(12,23) In contrast, other analyses of post-procedural DWI lesion volume following BAO have failed to demonstrate a consistent correlation with outcome. Possible reasons for this discrepancy include small sample size and the use of overall infarct volume alone, without accounting for pathoanatomic infarct distribution.(8)

Prior series evaluating reversal of $\mathrm{BAO}$ have demonstrated that the time window during which reperfusion continues to be beneficial is longer than in anterior circulation strokes.(13) A putative reason is that the brainstem may be more resistant to long-term ischemia than the cerebrum, including a more extensive network of collateral circulation and/or neuronal metabolism unique to the brainstem; which however remains unproven.(6) Regardless of mechanism, this suggests that a subset of well selected patients may continue to benefit from recanalization even at late time points. ${ }^{8}$

Our study has certain limitations. First, this is a retrospective study without a predetermined imaging inclusion/exclusion protocol. Patients included in this study were treated across 4 high-volume stroke centers in both the US and Europe, and therefore represent a broad, multinational spectrum of patients undergoing treatment for BAO. Second, numerically higher mortality rates in our derivation cohort

This article is protected by copyright. All rights reserved 
(44\%), compared to mortality rates in the BEST $(36 \%, p=0.19)$ and BASICS trials $(41 \%, p=0.46)$, may be explained by longer time to treatment in our patients. Third, outcomes were only analyzed at 3 months, which may be too early of a time point to fully capture eventual neurological recovery.(14) Fourth, the POST-VB score utilizes post-procedural scans, and therefore does not specifically address patient selection, but is instead limited to post-treatment prognostication. Nevertheless, this scoring system has significant clinical utility for prognostication, as even those patients destined for good recovery may still have poor clinical exams early after treatment when important decisions guiding post-procedural care are being made. Future studies will be necessary to address whether such information can be applied prior to continuation of intensive care to determine the likelihood of benefit.

\section{Conclusions}

This study demonstrates that the POST-VB scoring system can reliably predict outcome following acute reperfusion therapy for stroke due to BAO. This scoring system cannot replace diligent patient evaluation and comprehensive assessment. Nonetheless, the use of this grading system may help guide judicious patient selection for continued aggressive therapy following endovascular therapy and possibly decrease the risk inappropriately pursuing such care and avoid therapeutic futility. Importantly, the POST-VB demonstrated external validity across centers in both the US and Europe.

This article is protected by copyright. All rights reserved 


\section{REFERENCES}

1. Liu X, Dai Q, Ye R, Zi W, Liu Y, Wang H, et al. Endovascular treatment versus standard medical treatment for vertebrobasilar artery occlusion (BEST): an open-label, randomised controlled trial. Lancet Neurol. 2020 Feb;19(2):115-22.

2. Schonewille WJ, Algra A, Serena J, Molina CA, Kappelle LJ. Outcome in patients with basilar artery occlusion treated conventionally. J Neurol Neurosurg Psychiatry. 2005 Sep;76(9):1238-41.

3. Kang D-H, Jung C, Yoon W, Kim SK, Baek BH, Kim J-T, et al. Endovascular Thrombectomy for Acute Basilar Artery Occlusion: A Multicenter Retrospective Observational Study. J Am Heart Assoc. 2018 Jul 7;7(14).

4. Gory B, Eldesouky I, Sivan-Hoffmann R, Rabilloud M, Ong E, Riva R, et al. Outcomes of stent retriever thrombectomy in basilar artery occlusion: an observational study and systematic review. J Neurol Neurosurg Psychiatry. 2016 May;87(5):520-5.

5. Schonewille WJ, Wijman CA, Michel P, Rueckert CM, Weimar C, Mattle HP, et al. Treatment and outcomes of acute basilar artery occlusion in the Basilar Artery International Cooperation Study (BASICS): a prospective registry study. Lancet Neurol. 2009 Aug 1;8(8):724-30.

6. Lindsberg PJ, Mattle HP. Therapy of basilar artery occlusion: a systematic analysis comparing intraarterial and intravenous thrombolysis. Stroke. 2006 Mar;37(3):922-8.

7. Yoon W, Kim SK, Heo TW, Baek BH, Lee YY, Kang HK. Predictors of Good Outcome After StentRetriever Thrombectomy in Acute Basilar Artery Occlusion. Stroke. 2015 Oct;46(10):2972-5.

8. Bouslama M, Haussen DC, Aghaebrahim A, Grossberg JA, Walker G, Rangaraju S, et al. Predictors of Good Outcome After Endovascular Therapy for Vertebrobasilar Occlusion Stroke. Stroke. 2017;48(12):3252-7.

9. Strbian D, Sairanen T, Silvennoinen H, Salonen O, Kaste M, Lindsberg PJ. Thrombolysis of basilar artery occlusion: impact of baseline ischemia and time. Ann Neurol. 2013 Jun;73(6):688-94.

10. Puetz V, Sylaja PN, Coutts SB, Hill MD, Dzialowski I, Mueller P, et al. Extent of hypoattenuation on CT angiography source images predicts functional outcome in patients with basilar artery occlusion. Stroke. 2008 Sep;39(9):2485-90.

This article is protected by copyright. All rights reserved 
11. Schaefer PW, Yoo AJ, Bell D, Barak ER, Romero JM, Nogueira RG, et al. CT angiography-source image hypoattenuation predicts clinical outcome in posterior circulation strokes treated with intraarterial therapy. Stroke. 2008 Nov;39(11):3107-9.

12. Ostrem JL, Saver JL, Alger JR, Starkman S, Leary MC, Duckwiler G, et al. Acute basilar artery occlusion: diffusion-perfusion MRI characterization of tissue salvage in patients receiving intraarterial stroke therapies. Stroke. 2004 Feb;35(2):e30-34.

13. Linfante I, Llinas RH, Schlaug G, Chaves C, Warach S, Caplan LR. Diffusion-weighted imaging and National Institutes of Health Stroke Scale in the acute phase of posterior-circulation stroke. Arch Neurol. 2001 Apr;58(4):621-8.

14. Jung S, Mono M-L, Fischer U, Galimanis A, Findling O, De Marchis GM, et al. Three-month and longterm outcomes and their predictors in acute basilar artery occlusion treated with intra-arterial thrombolysis. Stroke. 2011 Jul;42(7):1946-51.

15. Singer OC, Berkefeld J, Nolte $\mathrm{CH}$, Bohner G, Haring H-P, Trenkler J, et al. Mechanical recanalization in basilar artery occlusion: the ENDOSTROKE study. Ann Neurol. 2015 Mar;77(3):415-24.

16. Zaidat OO, Yoo AJ, Khatri P, Tomsick TA, von Kummer R, Saver JL, et al. Recommendations on angiographic revascularization grading standards for acute ischemic stroke: a consensus statement. Stroke. 2013 Sep;44(9):2650-63.

17. Rangaraju S, Liggins JTP, Aghaebrahim A, Streib C, Sun C-H, Gupta R, et al. Pittsburgh outcomes after stroke thrombectomy score predicts outcomes after endovascular therapy for anterior circulation large vessel occlusions. Stroke. 2014 Aug;45(8):2298-304.

18. Mandrekar JN. Receiver Operating Characteristic Curve in Diagnostic Test Assessment. J Thorac Oncol. 2010 Sep 1;5(9):1315-6.

19. Arlot S, Celisse A. A survey of cross-validation procedures for model selection. Stat Surv. 2010;4:40-79.

20. Vickers AJ, Cronin AM, Elkin EB, Gonen M. Extensions to decision curve analysis, a novel method for evaluating diagnostic tests, prediction models and molecular markers. BMC Med Inform Decis Mak. 2008 Nov 26;8:53.

This article is protected by copyright. All rights reserved 
21. Vickers AJ, Elkin EB. Decision curve analysis: a novel method for evaluating prediction models. Med Decis Mak Int J Soc Med Decis Mak. 2006 Dec;26(6):565-74.

22. Liu X, Xu G, Zi W, Liu W, Zhou Z, Shi Z, et al. BASILAR ARTERY OCCLUSION ENDOVASCULAR INTERVENTION VERSUS STANDARD MEDICAL TREATMENT (BEST) TRIAL: PRIMARY RESULTS OF A MULTICENTER RANDOMIZED CONTROLLED TRIAL. In 2018.

23. Nagel S, Herweh C, Köhrmann M, Huttner HB, Poli S, Hartmann M, et al. MRI in patients with acute basilar artery occlusion - DWI lesion scoring is an independent predictor of outcome. Int J Stroke Off J Int Stroke Soc. 2012 Jun;7(4):282-8.

This article is protected by copyright. All rights reserved 
Table 1. Baseline Characteristics of Derivation Cohort (Center A, $n=59$ )

\begin{tabular}{|c|c|}
\hline Age (mean, years) & $64 \pm 13$ years \\
\hline Sex (male) & $34 \%(20)$ \\
\hline NIHSS Score (median) & 18 (IQR 22) \\
\hline Last known well to arrival (mean, hours) & 13 (IQR 35) \\
\hline \multicolumn{2}{|l|}{ Vascular Risk Factors } \\
\hline Hypertension & $59 \%(35)$ \\
\hline Diabetes Mellitus & $22 \%(13)$ \\
\hline Atrial Fibrillation & $19 \%(11)$ \\
\hline Hypertriglyceridemia & $39 \%(23)$ \\
\hline Coronary Artery Disease & $22 \%(13)$ \\
\hline Smoking & $22 \%(13)$ \\
\hline Intra-venous tPA & $12 \%(7)$ \\
\hline Intra-arterial thrombolytics & $42 \%(25)$ \\
\hline Endovascular thrombectomy & $85 \%(50)$ \\
\hline $\mathrm{TICl} 2 \mathrm{~B}$ or more & $90 \%(53)$ \\
\hline mRS $0-2$ at 90 days & $32 \%(19)$ \\
\hline mRS $0-3$ at 90 days & $39 \%(23)$ \\
\hline
\end{tabular}

This article is protected by copyright. All rights reserved 
Figure 1. Example of Volume Measurement by Infarct Location

Figure 2. Median Infarct Volume by Location

Figure 3. Receiver Operator Characteristic Curve for POST-VB Score at Different Sites

ROC- Receiver Operator Characteristic

Supplemental Material

Figure S1. Modified Rankin Scale Score at 3 months

Figure S2. Modified Rankin Scale Score at 90 days by binned POSTVB Score

mRS-modified Rankin Scale Score

This article is protected by copyright. All rights reserved 

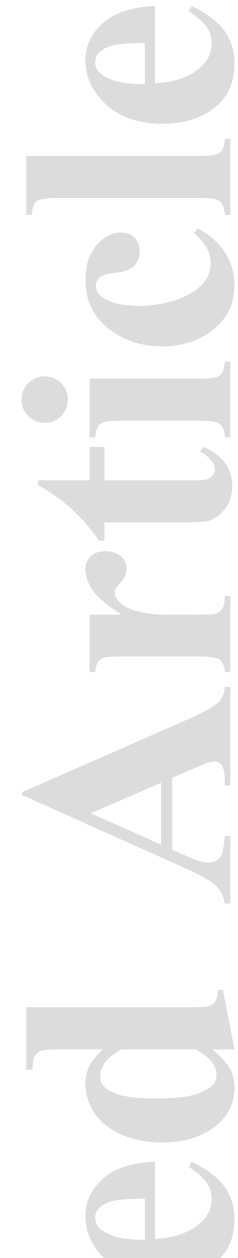

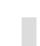
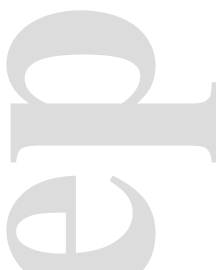<smiles>C1CC1</smiles>

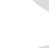<smiles>C1CCC1</smiles>
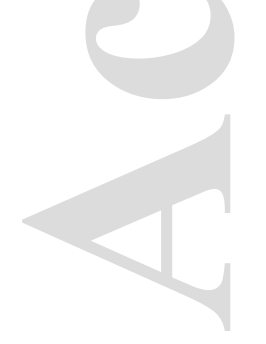

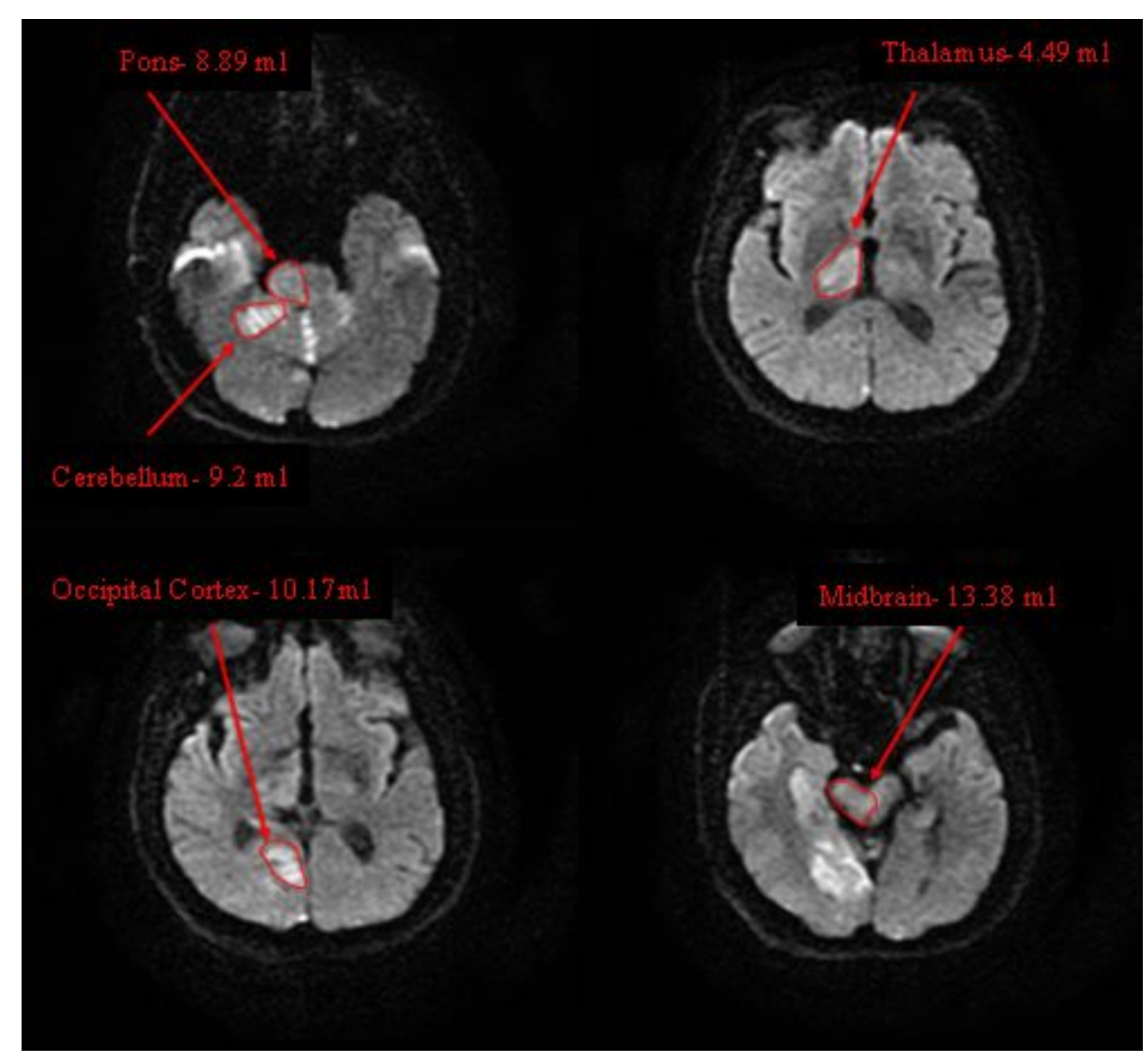

ene_14406_f1.jpg 


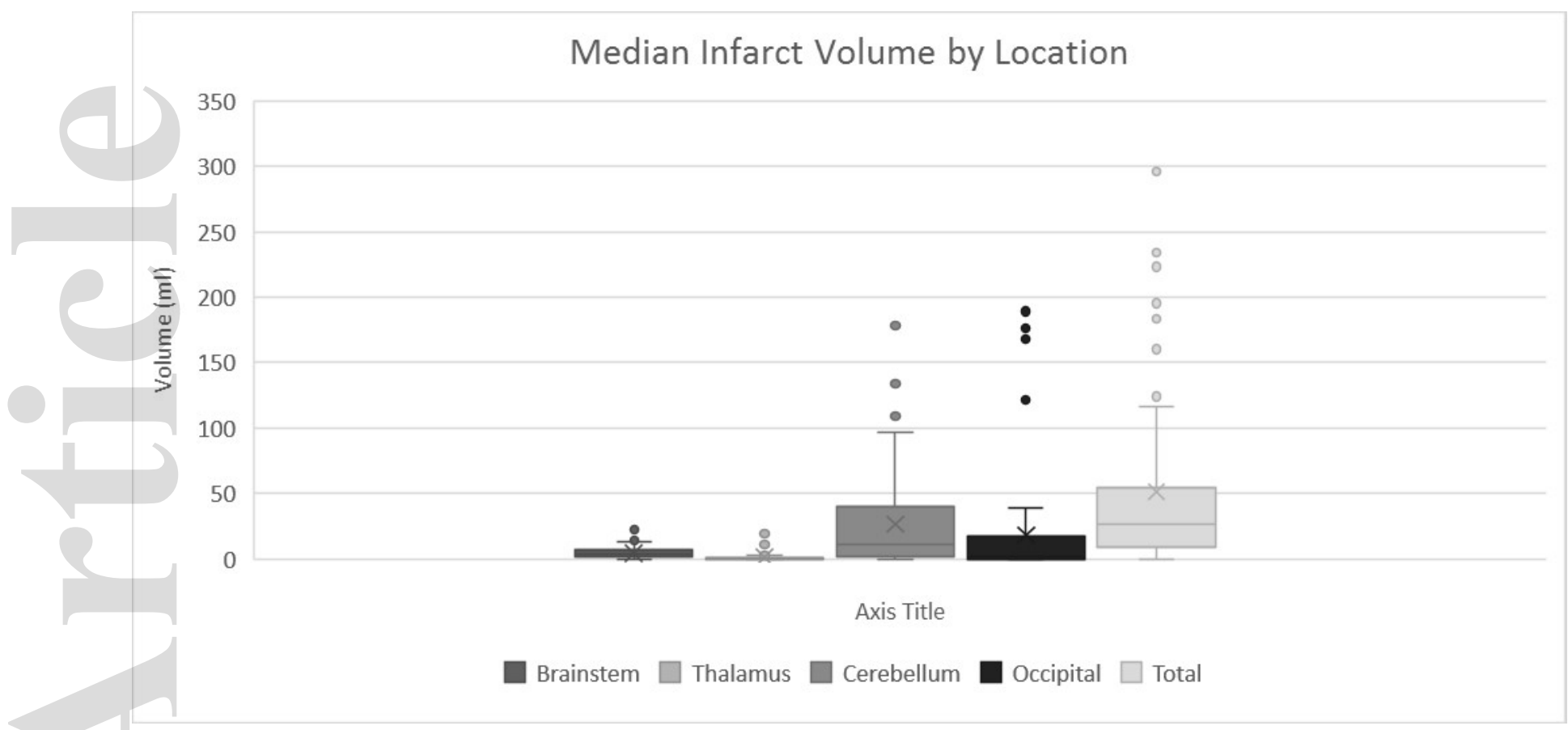

ene_14406_f2.jpg 

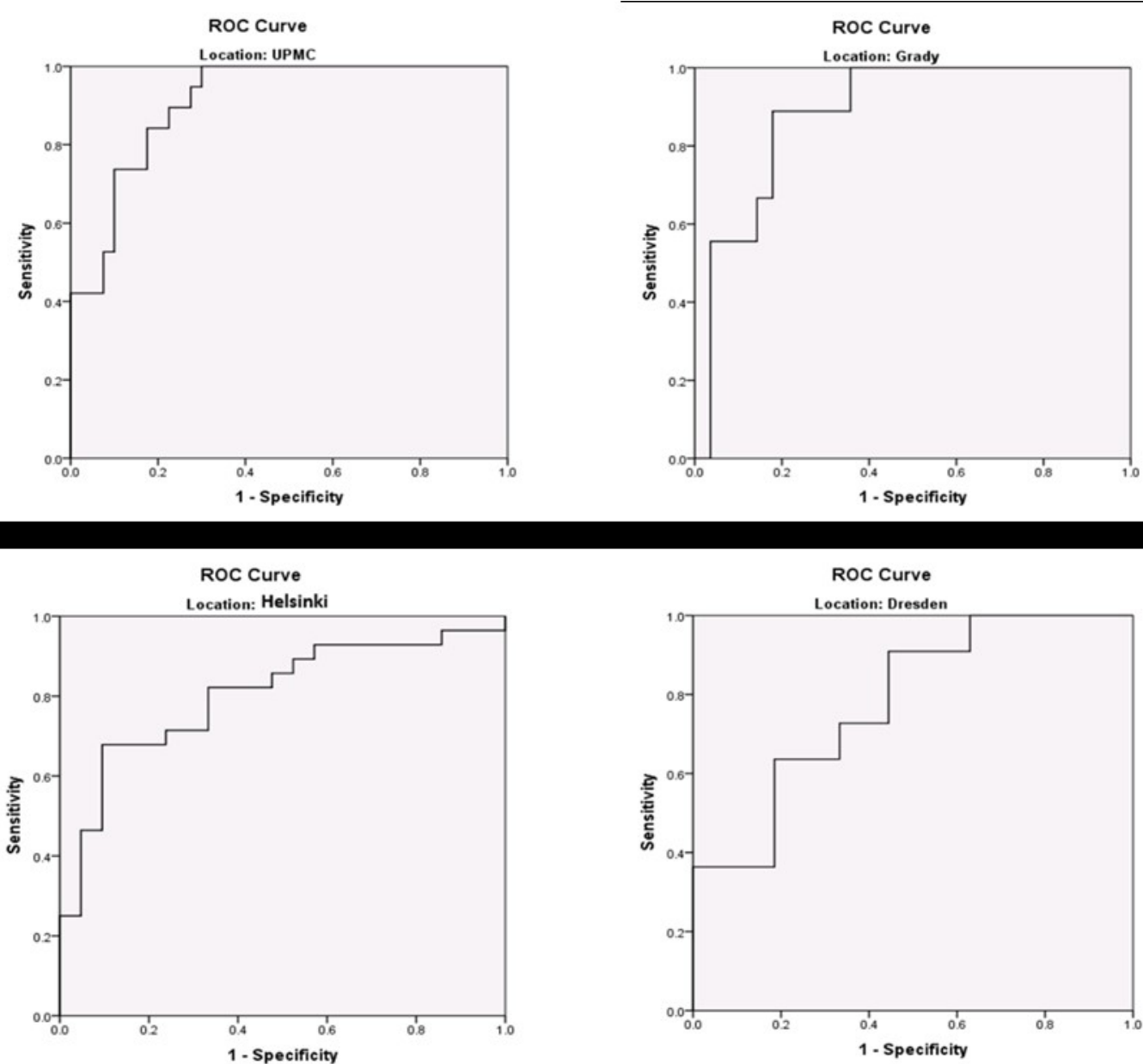

ene_14406_f3.jpg 